\title{
Prevalence of Haemoprotozoan diseases in crossbred cattle in Bangalore north
}

\author{
K. J. Ananda*, Placid E. D'Souza and G.C. Puttalakshmamma \\ Department of Veterinary Clinical Service Complex, \\ Veterinary College, Karnataka Veterinary, Animal and Fisheries Sciences University, Bangalore -
} 24

\begin{abstract}
The prevalence of haemoprotozoan disease in crossbred cattle was studied by screening 132 clinically suspected blood samples by Geimsa's stained blood smear method. Among 132 crossbred cattle screened, 57 animals were found positive for haemoprotozoan parasites. Out of 57 positive cases, 41 were found positive for Theileria annulata and the remaining 16 were positive for Babesia bibemina. The highest prevalence was found in 4-6 year age group and in monsoon months. The hematological observation revealed reduced $\mathrm{Hb}$, TEC and PCV in the infected animals. The treatment with buparvaquone and oxytetracyclin for theileriosis and diminazine aceturate for babesiosis are found effective.
\end{abstract}

Keywords: Prevalence, Theileria annulata, Babesia bibemina, Cattle, Bangalore north

\section{Introduction}

Haemoprotozoan diseases cause devastating losses to the livestock industry through out the world. However, it is known that most of blood protozoan parasites cause anemia by inducing erythrophagocytosis. Most of the haemoprotozoan parasites are tick borne and is of great economic importance in Asia and has always been a formidable barrier to the survival of exotic and cross bred cattle in India. Devendra, 1995 reported the annual loss of US \$ 800 million due to tropical theileriosis in India. Bovine babesiosis is also the most economically important disease because of direct losses of production. Many animals die and undergo a long period of convalescence entailing loss of meat and milk production. With early diagnosis and effective treatment, the mortality rate can be reduced. Hence in this context, an attempt was made to study the prevalence and treatment regimen for effective and early recovery from theileriosis and babesiosis in cross bred cattle in Bangalore North.

\section{Materials and Methods}

A total of 132 clinical cases of cross bred cattle with clinical symptoms such as anorexia, pyrexia, enlarged superficial lymph nodes, trembling in standing, pale conjuctival mucus membrane, haemoglobinuria, nasal discharge, coughing and grinding of teeth were screened for haemoprotozoan parasites. In addition to the above symptoms, there was sudden drop in the milk yield and four pregnant animals were aborted during the course of the disease were also examined for haemoprotozoan parasites.

Blood samples were collected with EDTA in a clean sterile tube from all 132 cross bred cattle. They were examined on the same day by Giemsa's stained blood smear. The hematological parameters such as hemoglobin, total erythrocyte count and packed cell volume were also recorded. The efficacy of the drugs includes Oxytetracyclin, Buparvaquone and Diminazine aceturate were studied.

\section{Results and Discussion}

Among 132 cattle screened by Giemsa's stainined blood smear, 57 were found positive for haemoprotozoan parasites. Out of 57 positive cases, 41 were found positive for Theileria annulata and the remaining 16 were positive for Babesia bibemina. Among 57 positive animals, 63.15\% were aged between 4-6 years, $21.05 \%$ between $1-2$ years and $15.79 \%$ were in below 6 months of age. This in accordance with Ruprah (1985) and Roy et al., (2004) who reported highest prevalence in animals aged more than 3 years followed by the lowest prevalence in less than one year age group.

The Hematological values were adversely affected in positive cases. The haemoglobin level was reduced to $8 \mathrm{gm} / 100 \mathrm{ml}$. In severely infected cases, it

* Corresponding author: E-mail-dranandakj@yahoo.co.in and mobile-09845313244 
Prevalence of Haemoprotozoan diseases in crossbred cattle in Bangalore north

was reduced to $3 \mathrm{gm} / 100 \mathrm{ml}$. The TEC and PCV were decreased to 2.3 million/cmm and $9 \%$ respectively. This might be due to damage caused by the organisms inside the RBC's during their multiplication. Mehta et al., (1988) found similar observations in experimentally induced cases of bovine tropical theileriosis. Similarly Muraleedharan, et al., (2005) reported low levels of haemoglobin and total erythrocyte counts in animals infected with Theileria annulata.

The study was conducted for one year to observe the seasonal prevalence, there was a considerable seasonal variation was found with the occurrence of haemoprotozoan disease in animals. Most of the animals suffered during monsoon months, which might be due to more number of ticks in monsoon which were developed during summer months. This is in accordance with the observations were made by Radostits et al., (1994) and Roy et al., (2004) they found highest prevalence in monsoon months.

The animals positive for theileriosis were treated with a single dose of buparvoquone @ 2.5mg/kg b.wt. $\mathrm{i} / \mathrm{m}$ (Butalex, Sarabhai) along with oxytetracyclin (Intamycin-LA, Intas Pharma, Ltd) @ 20 mg/kg b.wt. as single shot therapy. The animals positive for babesiosis were treated with diminazene aceturate @ 1gm/100kg b.wt. i/m (Berenil, Hoechst). The supportive treatment of Imferon-10ml, Belamyl-12ml and stadren-10ml i/m, were given for both the cases. Animals showed improvement in their condition after one week of the treatment.

\section{Acknowledgement}

The authors are thankful to Dr. P. Nangarajachar, Professor \& Head, (Rtd) Department of Veterinary Clinical Service Complex for giving permission and facility for conducting this work.

\section{References}

1. Devendra, C. (1995): In Global Agenda for Livestock Research, EDS, ILRI, Nairobi., pp 4148.

2. Mehta, H.K., Sisodia, R.S. and Misraulia, K.S. (1988): Indian J. Anim. Sci. 58(5):584-587.

3. Muraleedharan, K., K. Syed Ziauddin, P. Margoob Hussain, R. Puttabyatappa and S. J. Seshadri. (2005): J. Vet. Parasitol. 19(1): 71-72.

4. Radostits, O.M., Blood, D.C. and Gay, C.C. (1994): Veterinary Medicine, A text book of the diseases of cattle, sheep, Goats, Pigs and Horse, $8^{\text {th }}$ edn. ELBS, Baillier, London.

5. Roy, S., Tiwari, A., Galdhar, C.N., Upadhyay, S.R., Ratre, H.K., Sahu, S.K and Maiti, S.K. (2004): Indian J. Vet. Med. 24:5-7.

6. Ruprah, N.S. (1985): Text book of Clinical Protozoology, Oxanian Press Pvt. Ltd. New Delhi, pp. 286. 